\title{
RESSONÂNCIA MAGNÉTICA NUCLEAR DE PLATINA-195 EM COMPOSTOS ORGANOMETÁLICOS
}

\author{
Cleber Vinicius Ursini \\ Instituto de Química - Universidade Estadual de Campinas - CP 6154 - 13083-970 - Campinas - SP
}

Recebido em 5/3/96; aceito em 18/4/96

\begin{abstract}
PLATINUM-195 NUCLEAR MAGNETIC RESONANCE OF ORGANOMETALLIC COMPOUNDS. A brief review of ${ }^{195} \mathrm{Pt} \mathrm{NMR}$ is presented, focusing organometallic compounds. This article gives initially basic information of NMR processes involving ${ }^{195} \mathrm{Pt}$ nucleus. It is followed by a discussion of the factors which affect the chemical shifts and coupling constants. Finally, some aspects of ${ }^{195} \mathrm{Pt}$ NMR of solids are commented.
\end{abstract}

Keywords: NMR; ${ }^{195} \mathrm{Pt}$; platinum.

\section{INTRODUÇÃO}

Todos os processos químicos envolvendo a platina, desde o estudo de interações com o DNA até o seu encapsulamento em zeólitas, contam com o auxílio da técnica de Ressonância Magnética Nuclear de Platina-195 (RMN $\left.{ }^{195} \mathrm{Pt}\right)$. Durante os anos 60 , já era possível a obtenção de espectros de RMN ${ }^{195} \mathrm{Pt}$, apesar das limitações dos aparelhos. A partir dos anos 70, com o desenvolvimento da técnica de RMN de pulsos com transformada de Fourier, a RMN se desenvolveu rapidamente para vários núcleos ${ }^{1}$.

A RMN baseia-se nas transições entre níveis de energia de um núcleo com spin nuclear exposto a um campo magnético. A princípio, todos os núcleos com número quântico de spin nuclear $I$ diferente de zero, tais como ${ }^{1} \mathrm{H},{ }^{2} \mathrm{H},{ }^{10} \mathrm{~B},{ }^{31} \mathrm{P},{ }^{197} \mathrm{Au}$, etc, sofrem este fenômeno. Um spin nuclear se comporta como um dipolo magnético, que tende a se alinhar ao campo magnético aplicado. O magnetismo de um núcleo é descrito em termos de sua constante giromagnética $\gamma$, que é proporcional à razão entre o momento magnético nuclear $\mu$ e o momento angular de spin nuclear $I^{2}$ :

$\gamma=\mu / I(h / 2 \pi), \quad h=$ constante de Planck

Um núcleo ativo na RMN $(I \neq 0)$ é perturbado por um campo efetivo $\mathbf{B}_{\text {ef }} \mathbf{e}$ não pelo campo magnético $\mathbf{B}_{\mathbf{0}}$ diretamente. Este campo efetivo é resultado da soma do campo magnético originado da circulação dos elétrons em torno do núcleo (blindagem) com o campo aplicado $\mathbf{B}_{0}{ }^{2}$ :

$\mathbf{B}_{\text {ef }}=\mathbf{B}_{\mathbf{0}}(1-\sigma), \quad \sigma=$ blindagem magnética nuclear

A blindagem magnética nuclear $\sigma$ estabelece diferenças entre núcleos de mesma natureza em ambientes químicos diferentes.

$\mathrm{O}$ número quântico de spin nuclear $I$ define o número de níveis de energia $\mathrm{N}$ possíveis para um núcleo em um campo magnético através da relação:

$\mathrm{N}=(2 I+1)$

Assim, para $I=1 / 2$, característico de núcleos de ${ }^{1} \mathrm{H}$ e ${ }^{195} \mathrm{Pt}$, entre outros, existem dois níveis de energia (Fig. 1).

A diferença entre os níveis de energia, $\Delta E$, é diretamente proporcional a magnitude do campo magnético efetivo, situando-se na região de energia das radiofreqüências:

$\Delta E=(\gamma h / 2 \pi) \cdot B_{e f}=h v$

As populações de núcleos de spins paralelos e antiparalelos a $\mathbf{B}_{\mathbf{0}}$, nos níveis de energia, são praticamente iguais, com uma

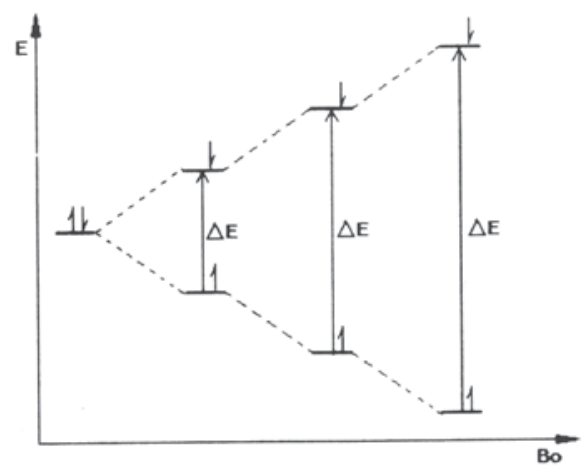

Figura 1. Desdobramento dos níveis dc energia para núcleos com $I=$ 1/2 em um campo magnético $\boldsymbol{B}_{\boldsymbol{0}}$.

pequena vantagem para o nível menos energético. Com o aumento do campo $\mathbf{B}_{\mathbf{0}}$, a diferença $\Delta E$ é ampliada (Fig. 1). Deste modo, o nível de energia menor tem um acréscimo na sua população de núcleos de spins paralelos a $\mathbf{B}_{0}{ }^{3}$.

$\mathrm{Na}$ técnica de RMN, o campo magnético de um ímã gera uma magnetização dos spins individuais que, somados, são responsáveis pela magnetização macroscópica $\mathbf{M}$, paralela ao campo $\mathbf{B}_{0}$ (Fig. 2a). Um sinal de radiofreqüência, responsável por um segundo campo magnético $\mathbf{B}_{\mathbf{1}}$, perpendicular ao campo $\mathbf{B}_{\mathbf{0}}$, varia continuamente dentro de uma faixa de freqüências $v$ (aparelhos de varredura contínua) ou é emitido na forma de um pulso contendo uma faixa de freqüências (aparelhos de pulso com transformada de Fourier). A magnetização total $\mathbf{M}$ sofre um torque e gira com uma certa inclinação em torno do eixo paralelo a $\mathbf{B}_{\mathbf{0}}$ (cone de precessão). Esta inclinação depende da potência e/ou do tempo de pulso da radiofrequiência. Quando a inclinação é $90^{\circ}$ (pulso de $90^{\circ}$ ), as populações de spins paralelos e antiparalelos ao campo $\mathbf{B}_{\mathbf{0}}$ estão igualadas (Fig. 2b). A variação de magnetização durante a relaxação dos núcleos é detectada no plano perpendicular a $\mathbf{B}_{\mathbf{0}}{ }^{\mathbf{3 , 4}}$. Os processos de relaxação que levam novamente o vetor magnetização $\mathbf{M}$ à posição de equilíbrio $\left(\mathbf{M}_{\mathbf{0}}\right)$ são divididos em duas classes: relaxação longitudinal, que é atribuída aos fenômenos que trazem de volta o vetor magnetização a sua posição inicial (paralelo a $\mathbf{B}_{\mathbf{0}}$ ), e a relaxação transversal, atribuída aos fenômenos que levam a desmagnetização no plano perpendicular a $\mathbf{B}_{\mathbf{0}}$ (Fig. 2). Estes dois processos de relaxação estão relacionados às duas constantes de tempo: $1 / T_{1}$ (relaxação longitudinal ou spin-rede) e $1 / T_{2}$ (relaxação transversal ou spin-spin) ${ }^{5}$. $\mathrm{O}$ valor de $T_{1}$ sempre e maior ou igual ao valor de $T_{2}$, pois a anulação das componentes $\mathbf{M}_{\mathbf{x}}$ e $\mathbf{M}_{\mathbf{y}}$ do vetor $\mathbf{M}$ (relaxação transversal) não depende de transferência de 
energia; basta que os spins individuais responsáveis por $\mathbf{M}$ tenham uma distribuição aleatória no cone de precessão (Fig. 2d). A relaxação longitudinal ou spin-rede ocorre com transferência de energia para outros graus de liberdade do meio sem emissão espontânea ${ }^{6}$ Em sólidos e líquidos viscosos, $T_{2}$ é menor que $T_{1}$ enquanto para amostras não viscosas, $T_{1}$ e $T_{2}$ são praticamente iguais ${ }^{7}$.
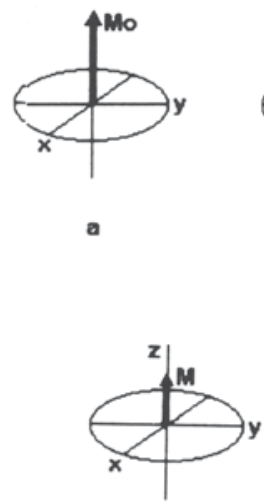

d

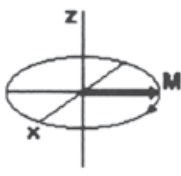

b

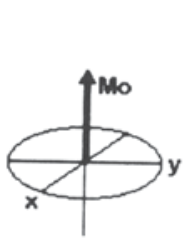

Figura 2. a) Representação do vetor $\boldsymbol{M}$ em um campo $\boldsymbol{B}_{0}$; b) logo depois de um pulso de $90^{\circ}$; c) durante os processos de relaxação; d) relaxação transversal completa $\left(T_{2}\right)$; e) relaxação longitudinal completa $\left(T_{1}\right)$.

Para núcleos com $I=1 / 2$, os valores de $T_{1}$ e $T_{2}$ são relativamente grandes, enquanto para núcleos com $I>1 / 2$, que apresentam momento de quadrupolo nuclear, $T_{1}$ e $T_{2}$ são pequenos. A interação de um momento de quadrupolo nuclear com um gradiente de campo elétrico proporciona um processo de relaxação muito eficiente ${ }^{7,8}$.

Os valores de $T_{1}$ para ${ }^{195} \mathrm{Pt}$ variam de 0,014 a $8,31 \mathrm{~s}$, embora a maioria deles esteja abaixo de $2 \mathrm{~s}$. Os mecanismos que mais contribuem para a relaxação deste núcleo são o de deslocamento químico anisotrópico e o de spin-rotação ${ }^{8,9}$.

$O$ resultado dos processos de magnetização e relaxação é convertido em um espectro, que apresenta linhas de ressonância em frequiências $v$ correspondentes aos valores de $\Delta E$ das transições dos núcleos estudados. Os valores de $v$ são proporcionais a $\mathbf{B}_{\mathbf{0}}$, portanto um determinado núcleo terá diferentes freqüências de ressonância em espectrômetros de diferentes valores de $\mathbf{B}_{\mathbf{0}}$. Para facilitar a análise do espectro, utiliza-se o deslocamento químico $\delta$ (Equação 5) ao invés da freqüência $v$. O deslocamento químico de um núcleo independe do campo $\mathrm{B}_{0}$.

$\delta=\left[\left(v_{\text {amostra }}-v_{\text {peferência }}\right) \cdot 10^{6}\right] / v_{\text {referência }}$

A utilização da técnica de RMN depende das propriedades inerentes a cada núcleo estudado. Estas propriedades são o número quântico de spin $I$, o momento de quadrupolo elétrico $Q$, a constante giromagnética $\gamma$ e a abundância natural $A$ do isótopo ativo. Núcleos com $I>1 / 2$ possuem momento de quadrupolo elétrico Q (distribuição elipsoidal de carga), que acelera os tempos de relaxação alargando os sinais. A "facilidade" de observação de um isótopo ativo na RMN depende da receptividade $R$ deste núcleo, que é proporcional a intensidade do sinal:

Intensidade do sinal $\propto R=\gamma^{3} A I(I+1)$

Para comparações, utiliza-se a receptividade relativa $R_{C}^{X}$ de um núcleo $(\mathrm{X})$ em relação ao núcleo de ${ }^{13} \mathrm{C}{ }^{2}$ :

$R^{X}{ }_{\mathrm{C}}=\left|\gamma_{\mathrm{X}}{ }^{3}\right| \cdot A_{\mathrm{X}} \cdot I_{\mathrm{X}} \cdot\left(I_{\mathrm{X}}+1\right) /\left|\gamma_{\mathrm{C}}{ }^{3}\right| \cdot A_{\mathrm{C}} \cdot I_{\mathrm{C}} \cdot\left(I_{\mathrm{C}}+1\right)$

A platina contém apenas um isótopo com spin nuclear: ${ }^{195} \mathrm{Pt}$. Os demais, com $I=0$, são: ${ }^{194} \mathrm{Pt}(A=32,9 \%),{ }^{196} \mathrm{Pt}(\mathrm{A}=25,3 \%)$ e ${ }^{198} \mathrm{Pt}(A=7,2 \%)^{0}$. 0 núcleo ${ }^{195} \mathrm{Pt}$ tem $R^{P t}{ }_{\mathrm{C}}=19,9$ e $I=1 / 2$ $(\text { Tabela } 1)^{11}$. Estas características colaboram para que este núcleo seja relativamente fácil de ser estudado por RMN.

Tabela 1. Algumas propriedades nucleares de ${ }^{1} \mathrm{H},{ }^{13} \mathrm{C}$ e ${ }^{195} \mathrm{Pt}$ (ref. 11)

\begin{tabular}{rccc}
\hline Núcleo & Abundância/\% & $\mathrm{R}^{\mathrm{Pt}} \mathrm{C}$ & $\gamma \cdot 10^{7} \mathrm{rad} / \mathrm{sT}$ \\
\hline${ }^{1} \mathrm{H}$ & 99,985 & $5,67.10^{3}$ & 26,7522 \\
${ }^{13} \mathrm{C}$ & 1,108 & 1,00 & 6,728 \\
${ }^{195} \mathrm{Pt}$ & 33,8 & 19,9 & 5,838 \\
\hline
\end{tabular}

\section{DESLOCAMENTOS QUÍMICOS EM RMN ${ }^{195}$ PT}

Os deslocamentos químicos estão relacionados com os fatores de blindagem magnética do núcleo $\sigma$. Quanto mais blindado ou protegido, menor será o deslocamento químico. O fator de blindagem magnética nuclear $\sigma$ para núcleos pesados como o núcleo de ${ }^{195} \mathrm{Pt}$ é dominado pela contribuição paramagnética $\sigma_{p}$, que deriva da circulação eletrônica diamagnética ao redor do núcleo de platina. A doação ou retirada de elétrons, através de substituintes ou ligantes na platina, modifica a distribuição eletrônica nos seus orbitais de valência causando alterações na contribuição paramagnética $\sigma_{p}{ }^{12}$.

Os valores de $\delta$ para núcleos de ${ }^{195} \mathrm{Pt}$ ocupam uma faixa muito ampla, aproximadamente $15.000 \mathrm{ppm}$, dificultando a localização do sinal. Por outro lado, é quase impossível que haja deslocamentos químicos iguais ou muito próximos para compostos diferentes ${ }^{1}$.

Uma solução $\mathrm{Na}_{2}\left[\mathrm{PtCl}_{6}\right]$ em água é a referência $(\delta=0)$ mais comum encontrada na literatura. Este sal é relativamente estável e disponível comercialmente. Porém, há alguns problemas como: a dependência de $\delta$ com a temperatura e com a concentração; a largura excessiva do sinal, devido aos acoplamentos entre ${ }^{195} \mathrm{Pt}$ e os isótopos ${ }^{35} \mathrm{C} 1$ e ${ }^{37} \mathrm{Cl}$ (ambos $I=3 / 2$ ), e a freqüência de ressonância muito alta em relação a maioria dos compostos de platina, o que leva a valores de $\delta$ negativos para estes compostos. A tabela 2 contém deslocamentos químicos de RMN ${ }^{195} \mathrm{Pt}$ para alguns complexos.

Outra alternativa de referência para valores de $\delta$ consiste em localizar $\delta=0$ exatamente na frequiência de $21,4 \mathrm{MHz}$ $\left[\Xi\left({ }^{195} \mathrm{Pt}\right)=21.400 .000 \mathrm{~Hz}\right]$ em uma "escala" onde os núcleos de ${ }^{1} \mathrm{H}$ do TMS (tetrametilsilano) sofrem ressonância exatamente a $100 \mathrm{MHz}^{13}$. Utilizando-se este modo de referência, o sinal de ressonância do sal $\mathrm{Na}_{2}\left[\mathrm{PtCl}_{6}\right]$ em $\mathrm{D}_{2} 0$ aparece em $\Xi\left({ }^{195} \mathrm{Pt}\right)$ $=21.496 .770 \mathrm{~Hz}$, o que equivale a $\delta(21,4 \mathrm{MHz})=+4522$ $\left(\delta\left(\left[\mathrm{PtCl}_{6}\right]^{2-}\right)=0\right)($ Equação 5$)$. Na literatura, os valores de $\delta$ são encontrados nas duas escalas, por isto a análise dos dados disponíveis deve ser feita cuidadosamente. Para a conversão entre as "escalas", utiliza-se a equação 8. Neste resumo, os valores de $\delta\left({ }^{195} \mathrm{Pt}\right)$ apresentados são relativos à ressonância do ânion $\left[\mathrm{PtCl}_{6}\right]^{2-}$ em 0 ppm.

$\delta\left(\left[\mathrm{PtCl}_{6}\right]^{2-}\right)=\delta(21,4 \mathrm{MHz})-4522$

Influência do estado de oxidação da platina nos valores de $\delta\left({ }^{195} \mathrm{Pt}\right)$

Os estados de oxidação comuns da platina são $4+, 2+$ e $0 . \mathrm{O}$ efeito generalizado da blindagem nuclear é maior para estados de oxidação menores. Complexos de Pt(IV) aparecem em freqüências mais altas, de $\mathrm{Pt}(\mathrm{II})$ em freqüências intermediárias e de $\operatorname{Pt}(0)$ em freqüências mais baixas (Fig. 3). Entretanto, há muitas exceções. Por exemplo, o composto organoplatina $\left[\mathrm{Pt}\left(\eta^{5}-\mathrm{C}_{5} \mathrm{H}_{5}\right) \mathrm{Me}_{3}\right]$ é, formalmente, um complexo de Pt(IV) com valor de $\delta$ típico de complexos de platina (0) $\left(-5275 \mathrm{ppm},-11,6^{\circ} \mathrm{C}, \mathrm{CDCl}_{3}\right)$. A ressonância do complexo de $\mathrm{Pt}(0),\left[\mathrm{Pt}(\operatorname{cod})_{2}\right]$ (cod $=1,5$-ciclooctadieno), aparece em frequiência mais alta: $\delta=-4636\left(-25^{\circ} \mathrm{C} \text {, tetraidrofurano- } d_{8}\right)^{17}$. 
Tabela 2. Deslocamentos químicos em RMN ${ }^{195} \mathrm{Pt}$ de alguns compostos de platina.

\begin{tabular}{|c|c|c|c|c|}
\hline complexo & estado de oxidação & $\delta$ & solvente & referência \\
\hline$\left[\mathrm{PtF}_{6}\right]^{2-}$ & $4+$ & +7325 & $\mathrm{CH}_{2} \mathrm{Cl}_{2}$ & 13 \\
\hline$\left[\mathrm{PtCI}_{6}\right]^{2-}$ & $4+$ & 0 & $\mathrm{D}_{2} \mathrm{O}$ & 9 \\
\hline$\left[\mathrm{PtBr}_{6}\right]^{2-}$ & $4+$ & -1894 & $\mathrm{D}_{2} \mathrm{O}$ & 9 \\
\hline$\left[\mathrm{PtI}_{6}\right]^{2-}$ & $4+$ & -6067 & $\mathrm{H}_{2} \mathrm{O}$ & 13 \\
\hline cis- $\left[\mathrm{Pt}\left(\mathrm{NH}_{3}\right)_{2} \mathrm{Cl}_{4}\right]$ & $4+$ & -145 & $\mathrm{H}_{2} \mathrm{O}$ & 9 \\
\hline $\mathrm{K}_{2}\left[\mathrm{Pt}(\mathrm{CN})_{6}\right]$ & $4+$ & -3866 & $\mathrm{D}_{2} \mathrm{O}$ & 9 \\
\hline$\left(\mathrm{NBu}_{4}\right)_{2}\left[\mathrm{Pt}(\mathrm{CN})_{6}\right]$ & $4+$ & -3321 & acetona- $d_{6}$ & 14 \\
\hline$\left[\mathrm{PtCl}_{4}\right]^{2-}$ & $2+$ & -1631 & $\mathrm{D}_{2} \mathrm{O}$ & 9 \\
\hline$\left[\mathrm{PtBr}_{4}\right]^{2-}$ & $2+$ & -2676 & $\mathrm{D}_{2} \mathrm{O}$ & 9 \\
\hline$c i s-\left[\mathrm{Pt}\left(\mathrm{NH}_{3}\right)_{2}\left(\mathrm{H}_{2} \mathrm{O}\right)_{2}\right]^{2+}$ & $2+$ & -1590 & $\mathrm{H}_{2} \mathrm{O}$ & 9 \\
\hline cis- $\left[\mathrm{Pt}\left(\mathrm{NH}_{3}\right)_{2} \mathrm{Cl}_{2}\right]^{2+}$ & $2+$ & -2168 & $\mathrm{H}_{2} \mathrm{O}$ & 9 \\
\hline cis-[Pt($\left.\left(\mathrm{PMe}_{3}\right)_{2} \mathrm{Cl}_{2}\right]$ & $2+$ & -4397 & $\mathrm{CD}_{3} \mathrm{NO}_{2}$ & 15 \\
\hline trans- $\left[\mathrm{Pt}\left(\mathrm{PMe}_{3}\right)_{2} \mathrm{Cl}_{2}\right]$ & $2+$ & -3939 & $\mathrm{CD}_{2} \mathrm{Cl}_{2}$ & 15 \\
\hline$\left[\mathrm{PtCl}_{2}(\operatorname{cod})\right]$ & $2+$ & -3351 & - & 16 \\
\hline$\left[\mathrm{PtMe}_{2}(\operatorname{cod})\right]$ & $2+$ & -3585 & - & 16 \\
\hline$\left[\mathrm{Pt}(\operatorname{cod})_{2}\right]$ & 0 & -4636 & thf- $d_{8}$ & 17 \\
\hline
\end{tabular}

Influência da temperatura, concentração e do solvente em $\delta\left({ }^{195} \mathbf{P t}\right)$

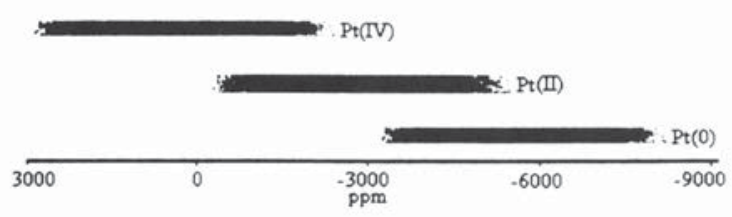

Figura 3. Faixas de deslocamentos químicos normalmente encontrados para compostos de platina (IV), (II) e (0).

Estudos da variação de $\delta$ com a temperatura, a concentração e o solvente para o complexo $\left[\mathrm{Pt}\left(\eta^{5}-\mathrm{C}_{5} \mathrm{H}_{5}\right) \mathrm{Me}_{3}\right]$ ilustram a preocupação com as condições da amostra em um experimento de RMN ${ }^{195} \mathrm{Pt}$. Uma alteração de temperatura na amostra, de $-11,6$ para $51,0^{\circ} \mathrm{C}$, resultou em uma variação de $\delta=-5257,2$ para $\delta$ $=-5219,5(\Delta \delta=37,7 \text {; Tabela } 3)^{18}$. Em geral, a variação de $\delta$ com a temperatura está na faixa de 0,5 a $1,1 \mathrm{ppm} / \mathrm{K}^{19 .}$ A influência da concentração nos valores de $\delta$ não é muito efetiva: uma variação de 0,056 para $2,024 \mathrm{M}$ a $22,0^{\circ} \mathrm{C}$ deslocou $\delta$ de 5237,6 para $-5235,7 \mathrm{ppm}(\Delta \delta=1,9 \text {; Tabela } 3)^{18}$. Em relação aos solventes da tabela 4 , a acetonitrila- $d$ e o clorofórmio- $d$ foram os que apresentaram a maior diferença entre os respectivos valores de $\delta(\Delta \delta=35)$.

Observando-se os valores de constantes dielétricas dos solventes da tabela 4 , não se nota nenhuma correlação da polaridade do solvente com o deslocamento químico. Outras interações entre o soluto e o solvente, além das dipolo-dipolo, também contribuem para a alteração do ambiente químico da platina.

\section{Efeito do isótopo}

$\mathrm{O}$ efeito do isótopo nos deslocamentos químicos $\delta\left({ }^{195} \mathrm{Pt}\right)$ é acentuado. Para o complexo $\left[\mathrm{Pt}\left(\eta^{5}-\mathrm{C}_{5} \mathrm{H}_{5}\right) \mathrm{Me}_{3}\right]$, quando os hidrogênios dos grupos metilas são trocados por deutérios, o valor de $\delta$ desloca-se para uma freqüência menor $(\Delta \delta=-36)(\text { Fig. } 4)^{18}$.
Tabela 3. Influência da temperatura e da concentração no $\delta\left({ }^{195} \mathrm{Pt}\right)$ do complexo $\left[\mathrm{Pt}\left(\eta^{5}-\mathrm{C}_{5} \mathrm{H}_{5}\right) \mathrm{Me}_{3}\right]$ em clorofórmio-d (ref. 18)

\begin{tabular}{ccc}
\hline concentração/M & temp. $/{ }^{\circ} \mathrm{C}$ & $\mathrm{ppm}$ \\
\hline 0,056 & 22,0 & -5238 \\
0,102 & 22,0 & -5237 \\
0,494 & 22,0 & -5237 \\
1,038 & 22,0 & -5236 \\
2,024 & 22,0 & -5236 \\
1,000 & $-11,6$ & -5257 \\
1,000 & 5,2 & -5247 \\
1,000 & 21,0 & -5237 \\
1,000 & 35,0 & -5230 \\
1,000 & 51,0 & -5220 \\
\hline
\end{tabular}

Tabela 4. Influência de alguns solventes no valor de $\delta\left({ }^{195} \mathrm{Pt}\right)$ do complexo $\left[\mathrm{Pt}\left(\eta^{5}-\mathrm{C}_{5} \mathrm{H}_{5}\right) \mathrm{Me}_{3}\right]\left(0,2 \mathrm{M}, 22,0^{\circ} \mathrm{C}\right)$ (ref. 18).

\begin{tabular}{lcc}
\hline solvente & constante dielétrica & ppm \\
\hline $\mathrm{C}_{6} \mathrm{D}_{12}$ & 2,02 & -5242 \\
$\mathrm{C}_{6} \mathrm{D}_{6}$ & 2,28 & -5252 \\
$\mathrm{CDCl}_{3}$ & 4,70 & 5237 \\
$\mathrm{C}_{5} \mathrm{D}_{5} \mathrm{~N}$ & 12,3 & -5260 \\
$\mathrm{CD}_{3} \mathrm{COCD}$ & 20,7 & -5269 \\
$\mathrm{CD}_{3} \mathrm{CN}$ & 36,2 & -5272 \\
$\mathrm{DMSO}_{3}$ & 49 & -5264 \\
\hline
\end{tabular}




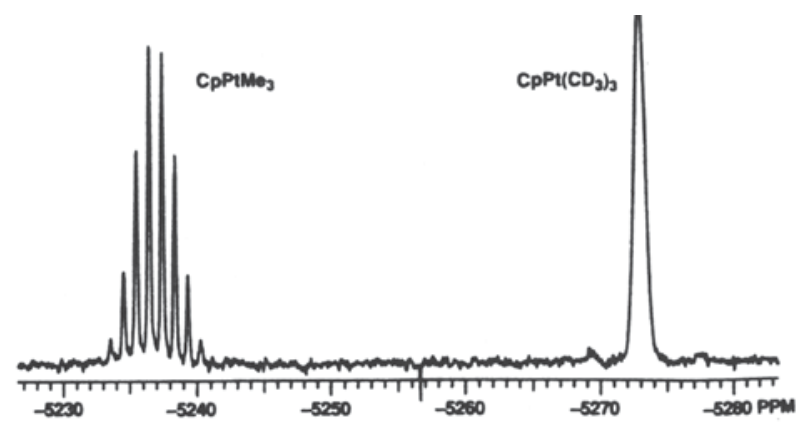

Figura 4. Efeito do isótopo no deslocamento químico: espectros de RMN ${ }^{195} \mathrm{PT}$ dos compostos $\left[\mathrm{Pt}\left(\eta^{5}-\mathrm{C}_{5} \mathrm{H}_{5}\right) \mathrm{Me}_{3}\right]$ e $\left[\mathrm{Pt}\left(\eta^{5}-\mathrm{C}_{5} \mathrm{H}_{5}\right) \mathrm{CD}_{3}\right]$ (ref. 18).

\section{Efeito do ligante e do número de coordenação}

$\mathrm{O}$ efeito do ligante sobre o valor de $\delta\left({ }^{195} \mathrm{Pt}\right)$ foi estudado para complexos do tipo $\left[\mathrm{PtCl}_{3} \mathrm{~L}\right]^{-}$. Para $\mathrm{L}=\mathrm{H}_{2} \mathrm{O}$ (um ligante duro), o valor de $\delta$ desloca-se para uma freqüência mais alta, $\delta=-1169$ (desprotegido), quando comparado aos valores de $\delta$ dos complexos com os ligantes moles $\mathrm{L}=\mathrm{SMe}_{2}(\delta=-2746) \mathrm{e}$ $\mathrm{PMe}_{3}(\delta=-3489)$. Ligantes moles coordenam-se mais fortemente à platina, deixando-a mais protegida em relação a ligantes duros como $\mathrm{H}_{2} \mathrm{O}$ e $\mathrm{Cl}^{-}$. $\mathrm{O}$ efeito também é bem pronunciado para os halogênios: complexos $\left[\mathrm{PtX}_{3}\left(\mathrm{AsMe}_{3}\right)\right]^{-}$apresentam deslocamentos químicos iguais a -3162, -3859 e -5435 ppm para $\mathrm{X}=\mathrm{Cl}$, Br e I, respectivamente ${ }^{9}$.

Complexos fosfinaplatina $(0),\left[\mathrm{Pt}\left(\mathrm{PR}_{3}\right)_{\mathrm{n}}\right]$, apresentam números de coordenação $n=2,3$ e 4 , com as respectivas geometrias linear, triangular e tetraédrica. Os valores de $\delta$ são menores para complexos bis-coordenados $\left(\delta\left[\mathrm{Pt}\left(\mathrm{Pcy}_{3}\right)_{2}\right]=-6555\right)$, intermediários para complexos tetracoordenados $\left(\delta\left[\mathrm{Pt}\left(\mathrm{PEt}_{3}\right) 4\right]=-\right.$ 5262) e maiores para complexos tricoordenados $\left(\delta\left[\mathrm{Pt}\left(\mathrm{PEt}_{3}\right)_{3}\right]\right.$ $=-4526$ e $\left.\delta\left[\mathrm{Pt}\left(\mathrm{Pcy}_{3}\right)_{3}\right]=-4567\right)^{20}$.

\section{CONSTANTES DE ACOPLAMENTOS DE ${ }^{195}$ PT}

O acoplamento escalar ou indireto $J$ entre dois núcleos ativos na RMN é transmitido através das ligações químicas que os separam ${ }^{21}$. Este é mais um fator de desdobramento dos níveis de energia de um núcleo com spin em um campo magnético. O diagrama de níveis de energia para dois núcleos ativos A e $\mathrm{B}$, ambos com $I=1 / 2$, mostra quatro transições possíveis: duas para o núcleo A e duas para o núcleo B (Fig. 5). Se $J(\mathrm{AB})=0$, o espectro de RMN resulta em dois singletos. Se $J(\mathrm{AB})>0$ ou $<0$, são observados dois dubletos, um para cada núcleo (Fig. 5$)^{5}$.

A expressão de contato de Fermi, para núcleos (A e B) separados por uma ligação, indica que ${ }^{1} J$ depende das constantes giromagnéticas dos núcleos envolvidos, $\gamma_{\mathrm{A}}$ e $\gamma_{\mathrm{B}}$, das densidades de elétrons dos orbitais s que participam da ligação, $|\Psi(0)|^{2}$, e da polarizabilidade mútua $\pi_{\mathrm{AB}}$, mas independem do campo magnético aplicado $\mathbf{B}_{0}{ }^{1}$ :

${ }^{1} J(\mathrm{~A}, \mathrm{~B}) \propto \gamma_{\mathrm{A}} \cdot \gamma_{\mathrm{B}} \cdot\left|\Psi(0)_{\mathrm{A}}\right|^{2} \cdot\left|\Psi(0)_{\mathrm{B}}\right|^{2} \cdot \pi_{\mathrm{AB}}$

A constante de acoplamento $J$ normalmente decresce com o aumento do número de ligações que separam os núcleos acoplados. Em muitos casos, os valores de $J$ envolvendo ${ }^{195} \mathrm{Pt}$ são suficientes para a caracterização de complexos. Estas constantes de acoplamento são medidas nos espectros de RMN ${ }^{195} \mathrm{Pt}$, como também nos espectros de núcleos acoplados aos núcleos de ${ }^{195} \mathrm{Pt}$. Por exemplo, a figura 6 apresenta um espectro de RMN ${ }^{31} \mathrm{P}$ desacoplado de ${ }^{1} \mathrm{H}$ (RMN $\left.{ }^{31} \mathrm{P}\left\{{ }^{1} \mathrm{H}\right\}\right)$ do complexo $\left[\mathrm{Pt}(\mathrm{dppf}) \mathrm{Cl}_{2}\right]\left[\mathrm{dppf}=1,1^{\prime}\right.$-bis(difenilfosfino)ferroceno], que contém dois fósforos quimicamente equivalentes (a abundância natural do núcleo de ${ }^{31} \mathrm{P}$ é praticamente de $\left.100 \%\right)$. Neste espectro,
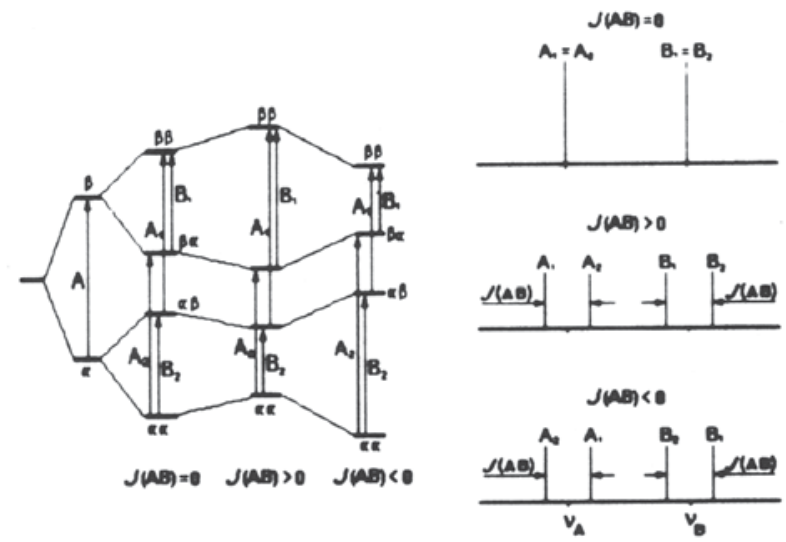

Figura 5. À esquerda: diagrama de níveis de energia para um sistema com dois núcleos A e B, ambos com spin 1/2. À direita: espectros de RMN com sinais originados das transições mostradas no diagrama de niveis de energia.

são observados um singleto central mais intenso, proveniente dos núcleos de ${ }^{31} \mathrm{P}$ de moléculas do isotopômero ${ }^{2}$ sem o isótopo ${ }^{195} \mathrm{pt}$ $(66,2 \%)$, e dois satélites (um dubleto), separados por ${ }^{1} J(\mathrm{PtP})=$ $3766 \mathrm{~Hz}$, resultantes das moléculas do isotopômero que contém núcleo de ${ }^{195} \mathrm{Pt}(33,8 \%)^{23}$.

Em RMN multinuclear, é comum a irradiação de núcleos de ${ }^{1} \mathrm{H}$, saturando-os. Este procedimento traz alguns benefícios: ganho na intensidade do sinal pelo efeito nuclear Overhauser, $\mathrm{NOE}^{24}$, e o desacoplamento de núcleos de ${ }^{1} \mathrm{H}$, que geralmente simplifica o espectro.

\section{RMN $M$ P $\{1 \mathbf{H}\}$}

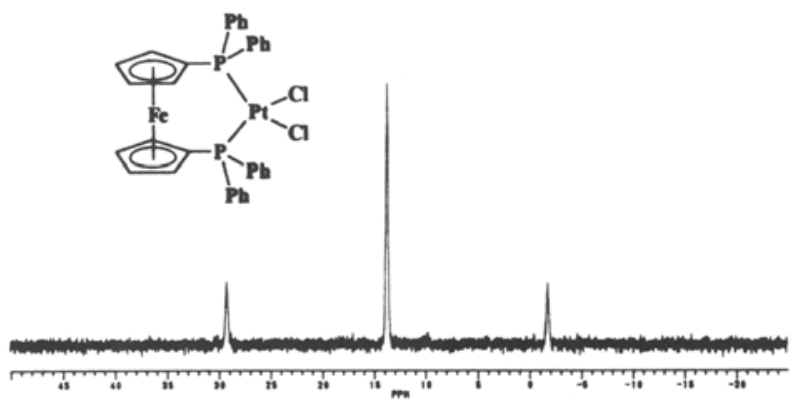

Figura 6. Espectro de $R M N{ }^{31} P\left\{{ }^{1} H\right\}$ do complexo [Pt(dppf)Cl $\left.\mathrm{Cl}_{2}\right]$.

É óbvio que os sinais observados em espectros de RMN ${ }^{195} \mathrm{Pt}$ são provenientes apenas de moléculas de isotopômeros que contêm um ou mais núcleos de ${ }^{195} \mathrm{Pt}$. A presença de outro núcleo ativo $(I \neq 0)$ na molécula, com abundância natural abaixo de $100 \%$, resulta em sinais satélites provenientes de acoplamentos deste núcleo com núcleos de ${ }^{195} \mathrm{Pt}$. Um bom exemplo é o espectro de $\mathrm{RMN}{ }^{195} \mathrm{Pt}\left\{{ }^{1} \mathrm{H}\right\}$ do complexo $\left[\mathrm{Pt}\left(\mathrm{SnCl}_{3}\right)_{3} \text { (norbornadieno) }\right]^{-}$(Fig. 7) ${ }^{9}$. O estanho tem dois isótopos ativos na RMN, ${ }^{117} \mathrm{Sn}$ e ${ }^{119} \mathrm{Sn}$, com abundâncias naturais de $7,61 \%$ e $8,58 \%$, respectivamente. Como há três átomos de estanho por molécula, a probabilidade de ocorrência de um dos isótopos ativos é três vezes maior que sua abundância natural. No espectro, existem dois conjuntos de satélites que são dois dubletos independentes. Os dubletos correspondem aos acoplamentos ${ }^{1} J\left({ }^{195} \mathrm{Pt}-{ }^{117} \mathrm{Sn}\right)$ e ${ }^{1} J\left({ }^{195} \mathrm{Pt}-{ }^{119} \mathrm{Sn}\right)$. O singleto central corresponde à ressonância de moléculas do isotopômero sem núcleo de ${ }^{117} \mathrm{Sn}$ ou ${ }^{119} \mathrm{Sn}$. Não são observados sinais de isotopômeros contendo ${ }^{195} \mathrm{Pt}$ juntamente com dois ou três núcleos de ${ }^{117} \mathrm{Sn}$ ou ${ }^{119} \mathrm{Sn}$. 
As abundâncias naturais destes isotopômeros são muito baixas para se destacarem do ruído do espectro.

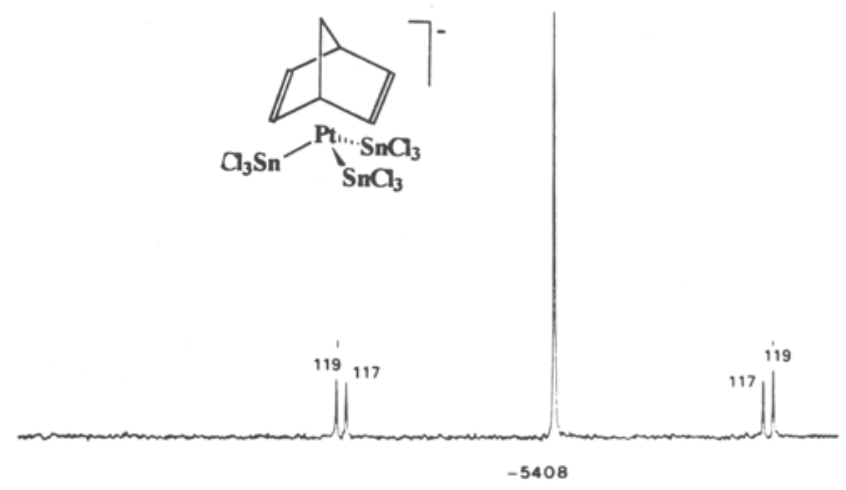

Figura 7. Espectro ele RMN ${ }^{195} \mathrm{Pt}\left[{ }^{1} \mathrm{H}\right]$ do ion-complexo $\left[\mathrm{Pt}\left(\mathrm{SnCl}_{3}\right)_{3}\right.$ (norbornadieno)J (ref. 9).

\section{Constantes de acoplamento $J(\mathrm{PtC}), J(\mathrm{PtH}), J(\mathrm{PtP})$ e $J(\mathrm{PtN})$}

Acoplamentos $J\left({ }^{195} \mathrm{Pt}^{13} \mathrm{C}\right)$ ou $J(\mathrm{PtC})$ em compostos organoplatina são geralmente observados em espectros de RMN ${ }^{13} \mathrm{C}\left\{{ }^{1} \mathrm{H}\right\}$. A baixa abundância natural do ${ }^{13} \mathrm{C}(\mathrm{A}=1,1 \%)$ dificulta a observação de $\mathrm{J}(\mathrm{PtC})$ em espectros de $\mathrm{RMN}{ }^{195} \mathrm{Pt}$. As ligações s entre os núcleos de ${ }^{195} \mathrm{Pt}$ e ${ }^{13} \mathrm{C}$ apresentam grandes contribuições de orbitais atômicos $2 s$ do carbono, resultando em acoplamentos $\mathrm{J}(\mathrm{PtC})$ relativamente altos $(700$ a $1500 \mathrm{~Hz}$ aproximadamente, dependendo dos orbitais híbridos que participam da ligação Pt-C). Ligações carbono-platina, envolvendo orbitais moleculares $\pi$ de olefinas ou acetilenos, apresentam ${ }^{1} \mathrm{~J}(\mathrm{PtC})$ bem menores $(10 \mathrm{a} 300 \mathrm{~Hz}$ aproximadamente, dependendo do envolvimento de orbitais $2 s$ dos carbonos ${ }^{25}$ ). $\mathrm{O}$ acoplamento de ${ }^{195} \mathrm{Pt}$ com um núcleo ativo $\mathrm{X}$ depende da natureza do ligante trans à ligação $\mathrm{Pt}-\mathrm{X}$. O complexo cis$\left[\mathrm{Pt}(\mathrm{C} \equiv \mathrm{CMe})_{2}(\mathrm{CO})\left(\mathrm{PMePh}_{2}\right)\right]\left(\right.$ Tabela 5) apresenta ${ }^{1} J(\mathrm{PtC}) \mathrm{da}$ alquinila trans à fosfina menor do que ${ }^{1} J(\mathrm{PtC})$ da alquinila trans à carbonila. Este fato é atribuído à maior influência trans ${ }^{26,27}$ da fosfina em relação à carbonila. Influência trans é a capacidade de um ligante enfraquecer a ligação oposta a ele (é um parâmetro termodinâmico, enquanto o efeito trans é um parâmetro cinético).

Acoplamentos ${ }^{1} J(\mathrm{PtH})$ em hidretos de platina variam em uma faixa de 700 a $1400 \mathrm{~Hz}$ aproximadamente ${ }^{31}$. Acoplamentos transmitidos por duas ligações, ${ }^{2} J(\mathrm{PtH})$, dependem do tipo de ligação entre o átomo de platina e o ligante que contém hidrogênio. Por exemplo, o complexo $\left[\mathrm{Pt}\left(\eta^{5}-\mathrm{C}_{5} \mathrm{H}_{5}\right)\left(\mathrm{CH}_{3}\right)_{3}\right]$ apresenta ${ }^{2} J(\mathrm{PtH})=$ $83 \mathrm{~Hz}$ para os núcleos de ${ }^{1} \mathrm{H}$ dos grupos metila (ligação $\mathrm{s}$ entre $\mathrm{Pt}$ e $\left.\mathrm{CH}_{3}\right)$ e ${ }^{2} J(\mathrm{PtH})=5,8 \mathrm{~Hz}$ para os núcleos de ${ }^{1} \mathrm{H}$ do grupo ciclopentadienfa (ligação $\pi$ entre $\mathrm{Pt}$ e $\left.\mathrm{C}_{5} \mathrm{H}_{5}\right)^{18}$.

Em relação ao núcleo ele ${ }^{31} \mathrm{P}$, os valores de ${ }^{1} J(\mathrm{PtP})$ variam dentro de um intervalo de $60 \mathrm{~Hz}$ a $9000 \mathrm{~Hz}$, aproximadamente. Foi observado que estes acoplamentos crescem com a diminuição do comprimento da ligação Pt-P e decrescem com o aumento da basicidade do fósforo, embora haja exceções. A contribuição de orbitais atômicos $3 s$ do fósforo na ligação Pt-P e a influência trans também são importantes para ${ }^{1} J(\mathrm{Ptp})^{32}$.

Os sinais de ressonância dos núcleos de ${ }^{195} \mathrm{Pt} \mathrm{em}$, complexos contendo ligação Pt-N costumam ser pouco resolvidos, dificultando uma boa visualização dos acoplamentos entre ${ }^{195} \mathrm{Pt}$ e ${ }^{14} \mathrm{~N}$ $(I=1)$. A relaxação quadrupolar do ${ }^{14} \mathrm{~N}$ é muito eficiente, o que reduz o tempo de relaxação dos núcleos de ${ }^{195} \mathrm{Pt}$, alargando seus sinais de ressonância. Em espectros de RMN ${ }^{195} \mathrm{Pt}$ de complexos contendo tiocianatos, cianatos e nitrilas como ligantes, é possível visualizar acoplamentos ${ }^{1} J\left({ }^{195} \mathrm{pt}-{ }^{14} \mathrm{~N}\right)$, que variam de 200 a $500 \mathrm{~Hz}$, conforme a influência trans $^{31}$. Espectros de RMN ${ }^{195} \mathrm{Pt}$ de complexos contendo ligantes nitrogenados são mais informativos, quando as amostras são enriquecidas com o isótopo ${ }^{15} \mathrm{~N}(I=1 / 2)$, que apresenta uma abundância natural muito baixa $(\mathrm{A}=0,37 \%)^{9}$.

\section{Relação entre $\mathbf{J}$ e a isomeria geométrica}

Os complexos isômeros de platina(IV) cis- e trans$\left[\mathrm{PtF}_{4} \mathrm{Cl}_{2}\right]^{2-}$ ilustram a relação entre a isomeria geométrica e as constantes de acoplamentos ${ }^{1} J\left({ }^{195} \mathrm{Pt}-{ }^{19} \mathrm{~F}\right)$. O complexo cis contém dois ligantes fluoros não equivalentes quimicamente, enquanto o trans possui todos os ligantes fluoros equivalentes quimicamente. Para o isômero trans, todos os quatro fluoros sofrem a mesma influência trans. Já o isômero cis apresenta dois ligantes cloros em posição trans a dois ligantes fluoros, o que resulta em constantes ${ }^{1} J(\mathrm{PtF})$ diferentes daquelas com

Tabela 5. Constantes de acoplamentos ${ }^{1} J\left({ }^{195} \mathrm{Pt}-{ }^{13} \mathrm{C}\right)$ para alguns complexos organoplatina.

\begin{tabular}{|c|c|c|c|}
\hline complexo & carbono acoplado & ${ }^{1} J\left(195 \mathrm{pt}-{ }^{13} \mathrm{C}\right) / \mathrm{Hz}$ & ref. \\
\hline \multirow[t]{3}{*}[\mathrm{Pt}(\eta^{5}-\mathrm{C}_{5}\mathrm{H}_{5})(\mathrm{COMe})\mathrm{Me}_{2}]{} & $\mathrm{CH}_{3}$ & 737 & 18 \\
\hline & $\underline{\mathrm{COMe}}$ & 999 & \\
\hline & $\eta^{5}-\underline{\mathrm{C}}_{5} \mathrm{H}_{5}$ & 15 & \\
\hline \multirow[t]{2}{*}[\mathrm{Pt}(\mathrm{CNBu}^{\mathrm{t}})_{2}(\eta^{2}-\mathrm{PhC}\equiv\mathrm{CPh})]{} & $\underline{\mathrm{CNBu}}^{\mathrm{t}}$ & 1415 & 28 \\
\hline & $\eta^{2}-\mathrm{Ph} \underline{\mathrm{C}} \equiv \underline{\mathrm{CPh}}$ & 332 & \\
\hline$\left[\mathrm{Pt}\left(\mathrm{PMe}_{3}\right)_{2}\left(\eta^{2}-\mathrm{PhC} \equiv \mathrm{CPh}\right)\right]$ & $\eta^{2}-\mathrm{PhC} \equiv \underline{\mathrm{C}} \mathrm{Ph}$ & 281 & 28 \\
\hline \multirow[t]{3}{*}{$c i s-\left\{\mathrm{Pt}(\mathrm{C} \equiv \mathrm{CMe})_{2}(\mathrm{CO})\left(\mathrm{PMePh}_{2}\right)\right]$} & $\underline{\mathrm{C}} \equiv \mathrm{CMe}$ trans $\mathrm{CO}$ & 1162 & 29 \\
\hline & $\underline{\mathrm{C}} \equiv \mathrm{CMe}$ cis $\mathrm{CO}$ & 1067 & \\
\hline & $\underline{\mathrm{CO}}$ & 1147 & \\
\hline$\left[\mathrm{Pt}(\operatorname{cod})_{2}\right]$ & $\eta^{2}-\underline{C}=\underline{C}$ & 143 & 17,30 \\
\hline$\left[\mathrm{Pt}(\mathrm{C} \equiv \mathrm{CPh})_{2}(\mathrm{dppf})\right]$ & $\underline{\mathrm{C}} \equiv \mathrm{CPh}$ & 1132 & 23 \\
\hline$\left[\mathrm{Pt}\left(\mathrm{C} \equiv \mathrm{CSiEt}_{3}\right)_{2}(\mathrm{dppf})\right]$ & $\underline{\mathrm{C}} \equiv \mathrm{CSiEt}_{3}$ & 1075 & 23 \\
\hline \multirow[t]{2}{*}{ cis $-\left[\mathrm{Pt}(\mathrm{C} \equiv \mathrm{CPh})_{2}\left(\mathrm{CNBu}^{\mathrm{t}}\right)_{2}\right]$} & $\underline{\mathrm{C}} \equiv \mathrm{CPh}$ & 1147 & 23 \\
\hline & $\mathrm{CNBu}^{\mathrm{t}}$ & 1051 & \\
\hline
\end{tabular}



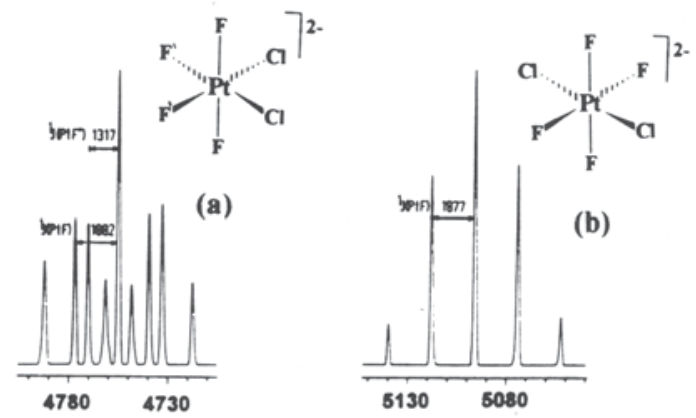

Figura 8. Espectro de RMN ${ }^{195} \mathrm{Pt}$ dos isômeros cis e trans $\left[\mathrm{PtF}_{4} \mathrm{Cl}_{2}\right]^{2-}$ (ref. 33).

\section{Influência da piramidalização de alquenos coordenados à platina}

A figura 9 mostra a estrutura básica de três complexos de platina (0): 1a, 1b e 1c, os quais contêm duas fosfinas e uma olefina cíclica coordenadas à platina. Nestes complexos, o tamanho da cadeia de grupos - $\mathrm{CH}_{2}$ - é alterado, permitindo diferentes ângulos de abertura para os dois anéis. Estes anéis têm em comum a olefina coordenada à platina. Quanto menor é o número de grupos $-\mathrm{CH}_{2}$ - separando os anéis, menor é o ângulo de abertura entre eles e maior é a piramidalização do alqueno. Isto afeta diretamente os valores de $\delta\left({ }^{195} \mathrm{Pt}\right),{ }^{1} J(\mathrm{PtC})$ e ${ }^{1} J(\mathrm{PtP})$ (Tabela 6). Uma maior piramidalização favorece a retrodoação do HOMO do fragmento [ $\left.\mathrm{Pt}\left(\mathrm{PPh}_{3}\right)_{2}\right]$ para o LUMO (orbital molecular $\left.\pi^{*}\right)$ do alqueno. $\mathrm{O}$ aumento da retrodoação tem o efeito sinérgico de aumentar a doação de elétrons do orbital $\pi$ da olefina para orbitais de mesma simetria na platina. Os valores de ${ }^{1} J(\mathrm{PtC})$ crescem por causa da interação mais forte da olefina com a platina (retrodoação e doação mais efetivas) e da maior contribuição de orbitais atômicos $2 s$ dos carbonos olefínicos na ligação

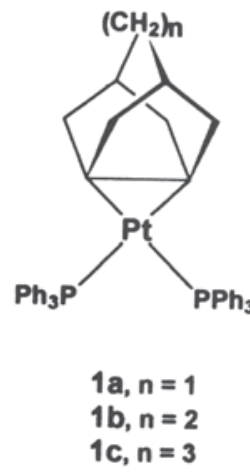

Figura 9. Estrutura basica dos complexos de platina (0) $\mathbf{1 a}, \mathbf{1 b}$ e $\mathbf{1 c}$, que contêm olefinas piramidalizadas.

Tabela 6. Deslocamentos químicos $\delta\left({ }^{195} \mathrm{Pt}\right)$ e constantes de acoplamento ${ }^{1} J(\mathrm{PtP})$ e ${ }^{1} J(\mathrm{PtC})$ dos complexos 1a, 1b, 1c e $\left[\mathrm{Pt}\left(\mathrm{Ph}_{3} \mathrm{P}\right)_{2}\left(\eta^{2}-C_{2} \mathrm{H}_{4}\right)\right]$ (ref. 25).

\begin{tabular}{lccc}
\hline complexo & $\delta$ & ${ }^{1} J(\mathrm{PtP}) / \mathrm{Hz}$ & ${ }^{1} J(\mathrm{PtC}) / \mathrm{Hz}$. \\
\hline 1a & -4989 & 2960 & 407 \\
1b & -5036 & 3115 & 343 \\
1c & -5023 & 3332 & 296 \\
{$\left[\mathrm{Pt}\left(\mathrm{Ph}_{3} \mathrm{P}\right)_{2}\left(\eta^{2}-C_{2} \mathrm{H}_{4}\right)\right]$} & -5077 & 3740 & 194 \\
\hline
\end{tabular}

do alqueno à platina. Conseqüentemente, as ligações Pt-P são enfraquecidas e os valores de ${ }^{1} J(\mathrm{PtP})$ reduzidos ${ }^{25}$.

\section{Complexos com mais de um átomo de platina}

O espectro de $\mathrm{RMN}{ }^{195} \mathrm{Pt}$ de um complexo com duas ou mais platinas é a soma ponderada dos espectros dos isotopômeros (subespectros) que contêm, ao menos, um núcleo de ${ }^{195} \mathrm{Pt}$. Cada isotopômero corresponde a um sistema de spin. Por exemplo, o complexo 2 possui praticamente quatro isotopômeros com os seguintes sistemas de spins: AA'BC $(43,8 \%)$, AA'BCM (22,4\%), AA'BCX $(22,4 \%)$ e AA'BCMX $(11,4 \%)$, nos quais $\mathrm{A}, \mathrm{A}^{\prime}, \mathrm{B}$ e $\mathrm{C}$ são núcleos de ${ }^{31} \mathrm{P}$, enquanto $\mathrm{X}$ e $\mathrm{M}$ são núcleos de ${ }^{195} \mathrm{Pt}$. O subespectro do sistema de spins AA'BC não apresenta linhas de ressonância no espectro de RMN 195Pt (não há núcleos de ${ }^{195} \mathrm{Pt}$ ). Os sistemas de spins restantes são responsáveis pelos seus respectivos subespectros, que somados fornecem o espectro experimental (Fig. 10a) ${ }^{34}$

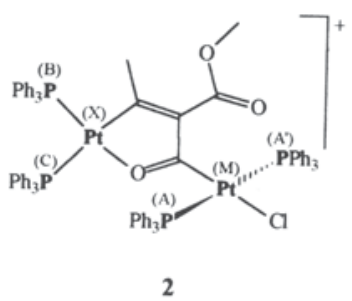

O sinal de ressonância do núcleo $\mathrm{Pt}(\mathrm{M})$ em -4061 ppm é um tripleto de dubletos (sistema de spins AA'BCM) com satélites (sistema de spins AA'BCMX), resultante dos seus acoplamentos com $\mathrm{P}(\mathrm{A}), \mathrm{P}\left(\mathrm{A}^{\prime}\right), \mathrm{P}(\mathrm{B})$ e $\mathrm{Pt}(\mathrm{X})$. Os núcleos $\mathrm{P}(\mathrm{A})$ e $\mathrm{P}\left(\mathrm{A}^{\prime}\right)$ são equivalentes quimicamente e apresentam constantes ${ }^{1} J(\mathrm{MA})=$ ${ }^{1} J\left(\mathrm{MA}^{\prime}\right)=2964 \mathrm{~Hz}$. O valor de ${ }^{4} J(\mathrm{MB})$ é de $127 \mathrm{~Hz}$, enquanto que, ${ }^{4} J(\mathrm{MC})$ não foi observado. O sinal do núcleo $\mathrm{Pt}(\mathrm{X})$ aparece em -4441 ppm. Este sinal é um dubleto de dubletos (sistema de spins AA'BCX) com satélites (sistema de spins AA'BCMX). Os núcleos $\mathrm{P}(\mathrm{B})$ e $\mathrm{P}(\mathrm{C})$ não são equivalentes quimicamente $\mathrm{e}$ acoplam ao núcleo $\mathrm{Pt}(\mathrm{X})$ com ${ }^{1} J(\mathrm{XB})=4100 \mathrm{~Hz}$ e ${ }^{1} J(\mathrm{XC})=$ $2057 \mathrm{~Hz}$. Acoplamentos ${ }^{4} J(\mathrm{XA})$ e ${ }^{4} J(\mathrm{XA}$ ') não foram observados. $\mathrm{O}$ posicionamento cis dos fósforos $\mathrm{A}, \mathrm{A}^{\prime}$ e $\mathrm{C}$ em relação à carbonila (em ponte com os átomos de platina) desfavorece a transmissão de acoplamentos ${ }^{4} J(\mathrm{PtP})$. O valor da constante de acoplamento entre os núcleos de ${ }^{195} \mathrm{Pt},{ }^{3} J(\mathrm{MX})=1000 \mathrm{~Hz}$, foi obtido pela simulação do sistema de spins AA'BCMX (Fig. 10e). Os sinais deste sistema correspondem aos picos satélites do espectro experimental (Fig.10a). Em conseqüência dos efeitos de segunda ordem, o valor de ${ }^{3} J(\mathrm{MX})$ não foi medido diretamente no espectro. A utilização de um aparelho de RMN mais potente (operando, por exemplo, em 42,8 MHz) evitaria os efeitos de segunda ordem, permitindo medidas diretas de deslocamentos químicos e de constantes de acoplamento no espectro, dispensando a simulação. Um espectro de RMN é considerado de primeira ordem, quando a diferença entre as frequiências de ressonâncias de dois núcleos é bem maior (pelo menos dez vezes maior) do que o acoplamento entre eles ${ }^{35}$ :

$|v(\mathrm{~A})-\mathrm{v}(\mathrm{B})| \gg J(\mathrm{AB})$

No espectro da figura 10a, a diferença $|v(M)-v(X)|$ é de $6540 \mathrm{~Hz}$, enquanto ${ }^{4} J(\mathrm{MX})$ é igual a $1000 \mathrm{~Hz}$; ou seja, $\mid v(\mathrm{M})$ - $v(\mathrm{X}) \mid$ é aproximadamente seis vezes maior do que ${ }^{4} J(\mathrm{MX})$ (espectrômetro operando em 17,2 MHz). Em um espectro obtido por um aparelho operando na freqüência de $42,8 \mathrm{MHz}$, a diferença $|v(M)-v(X)|$ seria igual a $16260 \mathrm{~Hz}$, que é aproximadamente dezesseis vezes maior do que ${ }^{4} J(\mathrm{MX})$. No entanto, espectros em aparelhos com campos magnéticos mais fortes podem ser prejudicados pelo aumento do efeito de deslocamento 
RMN ${ }^{196 P t}\left\{{ }^{1} \mathrm{H}\right\}$
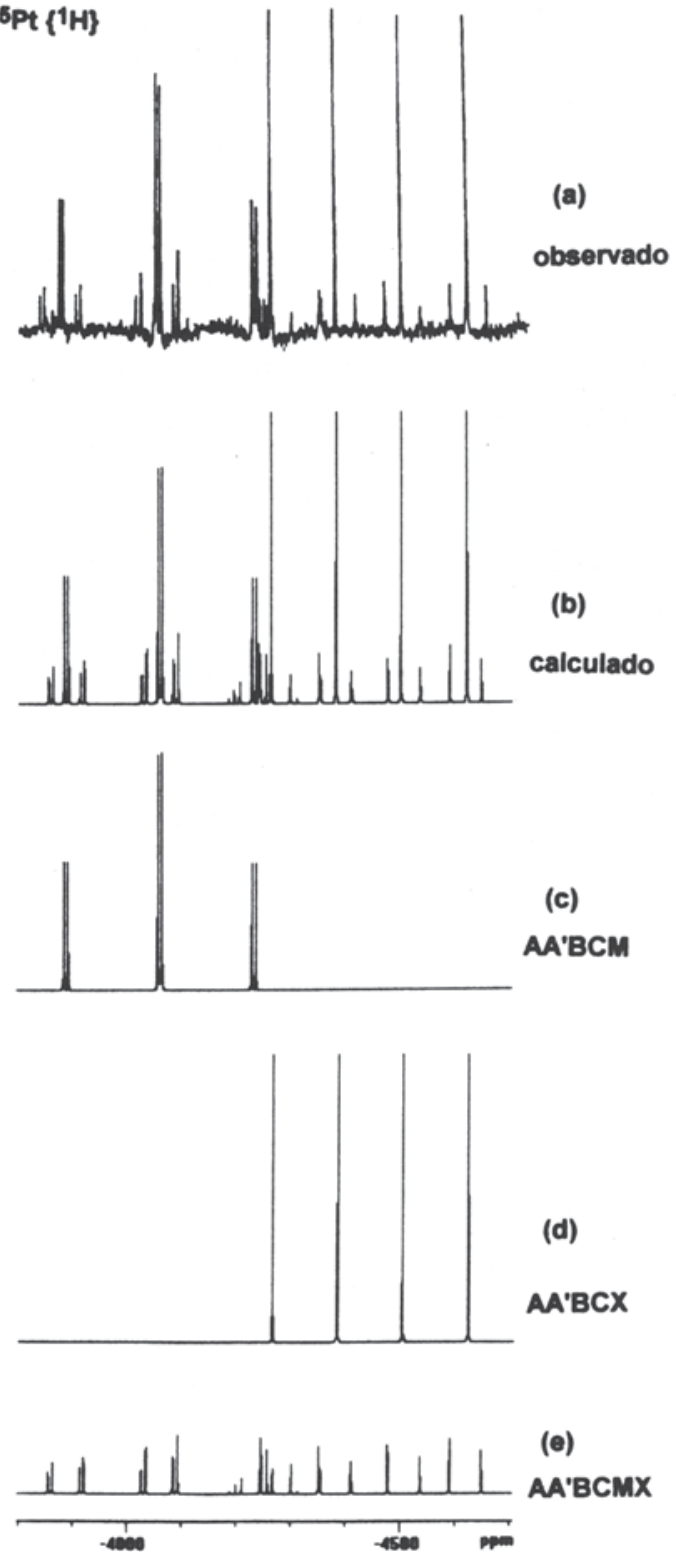

Figura 10. Estudos de RMN $\left.{ }^{195} \mathrm{Pt}^{1}{ }^{1} \mathrm{H}\right\}$ do composto 2. a) Espectro experimental (ref. 34); b) espectro simulado; c) subespectro simulado do sistemas spin $A A^{\prime} B C$; d) subespectro simulado do sistemas de spin $A A^{\prime} B C X$; e) subespectro simulado do sistemas de spin AA'BCMX. Freqüência de operação: 17,2 $\mathrm{MHz}$.

químico anisotrópico, que gera uma relaxação spin-rede mais efetiva, alargando os sinais ${ }^{36}$.

\section{Complexos com ligação Pt-Pt}

Os complexos dinucleados $\left[\mathrm{Pt}_{2} \mathrm{X}_{2}(\mu-\mathrm{dppm})_{2}\right][\mathrm{X}=\mathrm{Cl}$ (3a), $\operatorname{Br}(\mathbf{3 b}), \mathbf{I}(\mathbf{3 c})$ e dppm = bis(difenilfosfino)metano] contêm ligações metal-metal, cujos valores de ${ }^{1} J(\mathrm{PtPt})$ estão na faixa de 8000 a $9000 \mathrm{~Hz}$, aproximadamente (Fig. 11). O subespectro resultante do sistema de spin AA'A"A"'XX' $\left(A={ }^{31} \mathrm{P}, \mathrm{X}={ }^{195} \mathrm{Pt}\right)$ é de segunda ordem, o que impossibilita a medida de ${ }^{1} J(\mathrm{PtPt})$ diretamente no espectro ${ }^{37}$.

Acoplamentos entre núcleos de platina separados por uma ligação não são obrigatoriamente grandes (acima de $5000 \mathrm{~Hz}$ ). Acoplamentos abaixo de $1000 \mathrm{~Hz}$ ou mesmo com sinais negativos são encontrados em clusters triangulares de platina. $\mathrm{O}$ acoplamento ${ }^{1} J(\mathrm{PtPt})$ para o triângulo $\left[\mathrm{Pt} 3\left(\mu-\mathrm{CNBu}^{\mathrm{t}}\right)_{3}\left(\mathrm{CNBu}^{\mathrm{t}}\right)_{3}\right]$ (4), por exemplo, é de $188 \mathrm{~Hz}$ em $\mathrm{CD}_{2} \mathrm{Cl}_{2}{ }^{38}$, enquanto o

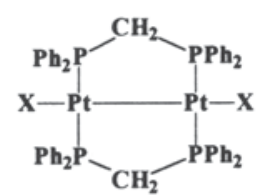

$$
\begin{aligned}
& \text { 3a } \mathrm{X}=\mathrm{Cl}, J(\mathrm{PtPt})=8145 \mathrm{~Hz} \\
& \text { 3b } \mathrm{X}=\mathrm{Br}, J(\mathrm{PtPt})=8805 \mathrm{~Hz} \\
& \text { 3c } \mathrm{X}=\mathrm{I}, J(\mathrm{PtPt})=9007 \mathrm{~Hz}
\end{aligned}
$$

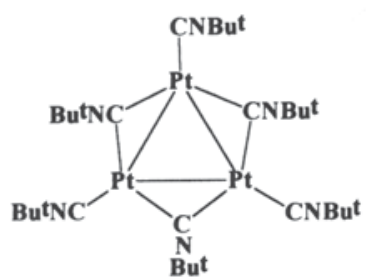

$J(\mathrm{PtPt})=188 \mathrm{~Hz}$

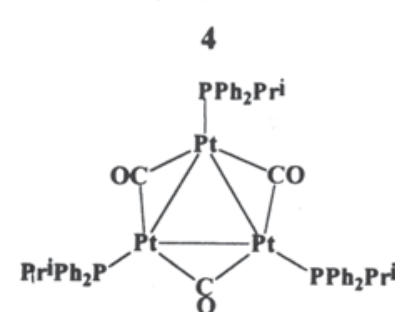

$J(\mathrm{PtPt})=-1667 \mathrm{~Hz}$

5

Figura 11. Complexos contendo ligações platina-platina.

complexo $\left[\mathrm{Pt}_{3}(\mu-\mathrm{CO})_{3}\left(\mathrm{PPh}_{2} \mathrm{Pr}^{\mathrm{i}}\right)_{3}\right] \quad$ (5) apresenta um acoplamento de $-1667 \mathrm{~Hz}^{39}$. Os acoplamentos ${ }^{1} J(\mathrm{PtPt})$ em complexos deste tipo, na verdade, são resultantes de várias possibilidades de acoplamentos transmitidos por diferentes caminhos através das ligações químicas. Por exemplo, o acoplamento ${ }^{1} J(\mathrm{PtPt})$ no complexo $\mathbf{3 a}$ é transmitido através da única ligação separando os dois átomos de platina, ${ }^{1} J(\mathrm{Pt}-\mathrm{Pt})$, e através das ligações dos ligantes dppm em ponte, ${ }^{4} J(\mathrm{Pt}-\mathrm{P}-$ C-P-Pt). Os complexos $\mathbf{4}$ e $\mathbf{5}$ permitem contribuições de acoplamentos ${ }^{1} J(\mathrm{Pt}-\mathrm{Pt}),{ }^{2} J(\mathrm{Pt}-\mathrm{C}-\mathrm{Pt})$ e ${ }^{2} J(\mathrm{Pt}-\mathrm{Pt}-\mathrm{Pt})$, entre outras de menor importância. Para o complexo 5, especificamente, foi determinado que o sinal relativo de seu acoplamento ${ }^{1} J(\mathrm{PtPt})$ é negativo $(-1667 \mathrm{~Hz})$. Isto significa que a soma das contribuições de acoplamentos ${ }^{2} J(\mathrm{Pt}-\mathrm{C}-\mathrm{Pt})$ e ${ }^{2} \mathrm{~J}(\mathrm{Pt}-\mathrm{Pt}-\mathrm{Pt})$, que são negativos, superam a contribuição do acoplamento ${ }^{1} J(\mathrm{Pt}-\mathrm{Pt})$, que é positivo ${ }^{39}$.

Acoplamentos ${ }^{1} J(\mathrm{PtPt})$ também podem ser obtidos em espectros de RMN de núcleos que não sejam ${ }^{195} \mathrm{Pt}{ }^{31}$. O espectro de RMN ${ }^{3 l} \mathrm{P}\left\{{ }^{1} \mathrm{H}\right)$ do complexo $\left[\mathrm{Pt}_{2} \mathrm{Fe}\left(\mu \text {-dppm)- }\left(\mathrm{CNBu}^{\mathrm{t}}\right)_{2}(\mathrm{CO})_{4}\right]^{5}\right.$ (6) (Fig. 12) traz esta informação através do subespectro de moléculas do isotopômero que contém duas platinas ativas. Este isotopômero apresenta o sistema de spins AA'XX' com abundância natural de $11,4 \%$ ( $A, A^{\prime}={ }^{31} p$ e $\left.X, X^{\prime}={ }^{195} \mathrm{Pt}\right)$. O subespectro mostra seis sinais. Dois deles são os satélites difusos próximos do sinal central $\left(a, a^{\prime}\right)$. Outros dois sinais, também largos e de baixa intensidade, estão localizados externamente $(b$, $\left.b^{\prime}\right)$. Finalmente, há dois picos relativamente finos e intensos $(c$, $c^{\prime}$ ) situados em frequiências intermediárias às dos sinais $a$ e $b$ e às dos sinais $a^{\prime}$ e $b^{\prime}$ (Fig. 12). Contudo, todos estes sinais são menos intensos do que aqueles provenientes dos sistemas de spins AA' $(A=43,8 \%)$ e AA'X $(A=44,8 \%)$, cujos isotopômeros são mais abundantes.

Em sistemas de spins $\mathrm{AA}^{\prime} \mathrm{XX}$ ' são permitidas 24 transições de intensidades significativas, mas, por causa da sobreposição de algumas delas, são observadas somente 10 linhas próximas 
RMN IIP $\{\mathbf{H}\}$

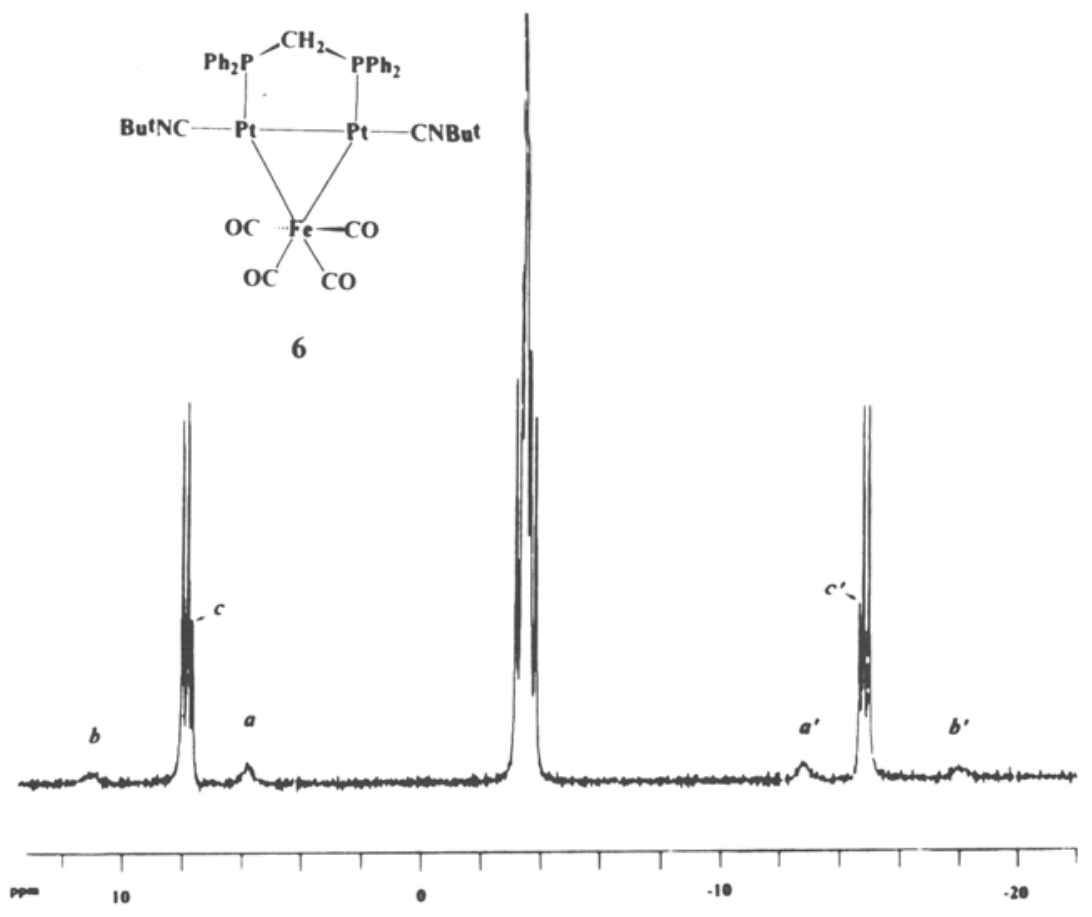

Figura 12. Espectro de $\mathrm{RMN}{ }^{31} \mathrm{Pt}\left\{{ }^{1} \mathrm{H}\right\}$ do complexo $\left[\mathrm{Pt}_{2} \mathrm{Fe}(\mu-\mathrm{dppm})\left(\mathrm{CNBu}{ }^{t}\right)_{2}(\mathrm{CO})_{4}\right]$ (6) em $\mathrm{CD}_{2} \mathrm{Cl}_{2}$. Freqüência de operação: 162,0 MHz.

de $v_{\mathrm{A}}$ e 10 linhas próximas de $v_{\mathrm{X}}$. $\mathrm{O}$ espectro de núcleos $\mathrm{A}$ é idêntico ao espectro de núcleos X; assim, as ressonâncias de A não fornecem nenhuma informação adicional do sistema de spins AA'XX' além daquelas que são obtidas pelas ressonâncias de $\mathrm{X}$ e vice-versa ${ }^{41}$. Na prática, a análise pode ser feita tanto pelo espectro de A como pelo de $\mathrm{X}$, que são idênticos. Muitas vezes, é possível obter somente um espectro: de A ou de X, ou então, são obtidos os dois espectros, mas um deles apresenta uma melhor resolução permitindo uma análise mais apurada.

As freqüências das linhas de ressonância de $\mathrm{A}$ ou de $\mathrm{X}$ no sistema $\mathrm{AA}^{\prime} \mathrm{XX}$ ' são dadas em termos dos parâmetros $K, L$, $M, N, P$ e $R$. Estes parâmetros são definidos a seguir ${ }^{41}$ :

$K=J\left(\mathrm{AA}^{\prime}\right)+J\left(\mathrm{XX}^{\prime}\right)$

$\mathrm{L}=J(A X)-J\left(A X^{\prime}\right)$

$M=J\left(A A^{\prime}\right)-J\left(\mathrm{XX}^{\prime}\right)$

$N=J(A X)+J\left(A X^{\prime}\right)$

$2 P=(K 2+L 2) 1 / 2$

$2 R=\left(M 2+L^{2}\right)^{1 / 2}$

O valor da constante de acoplamento $J\left(X^{\prime}\right)$ é igual a $(K$ $M) / 2$ e o módulo de $J\left(\mathrm{XX}^{\prime}\right)$ é igual a média entre os módulos de $K$ e de $M$, independentemente dos sinais relativos de $J\left(\mathrm{AA}^{\prime}\right)$ e de $J\left(X^{\prime}\right)$ :

$\left|J\left(\mathrm{XX}^{\prime}\right)\right|=(|K|+|M|) / 2$

Os módulos de $K, M$ e $\mathrm{N}$ e os valores de $2 P$ e $2 R$ correspondem às separações entre linhas de ressonância de $\mathrm{A}$ ou de $\mathrm{X}$ no espectro do sistema de spins AA'XX' (Fig. 13). Dependendo dos sinais relativos das constantes de acoplamento $J\left(\mathrm{AA}^{\prime}\right)$ e $J\left(\mathrm{XX}^{\prime}\right)$, o módulo de $K$ pode ser maior do que o módulo de $M$ ou vice-versa. As separações entre linhas, correspondentes aos módulos de $K$ e de $M$, são indistinguíveis ao analisarmos o espectro, o que não permite a obtenção dos sinais relativos de $J\left(\mathrm{AA}^{\prime}\right)$ e $J(\mathrm{XX})$. Voltando ao espectro de $\mathrm{RMN}{ }^{31} \mathrm{P}\left\{{ }^{1} \mathrm{H}\right\}$ do complexo 6 (Fig. 12), concluiu-se que cada um dos pequenos e largos sinais $\left(a, a^{\prime}, b\right.$ e $\left.b^{\prime}\right)$ contém duas linhas de ressonância, de acordo com o espectro simulado (Fig. 13). A distância, em Hz, entre o centro do sinal $a$ e o centro do sinal $b$, como também a distância entre o centro do sinal $a^{\prime}$ e o centro do sinal $b^{\prime}$, correspondem a $\left|J\left(\mathrm{XX}^{\prime}\right)\right|$ ou $|J(\mathrm{PtPt})|$, que é igual a média entre $|K|$ e $|M|$. A constante $|J(\mathrm{PtPt})|$ obtida foi de $(850 \pm 10) \mathrm{Hz}$.

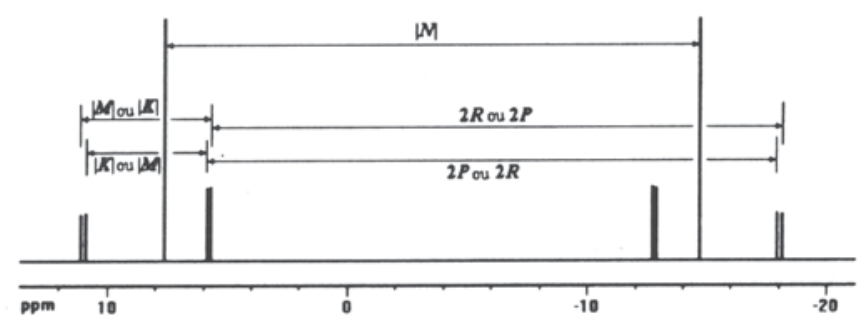

Figura 13. Simulação da parte A do sistema de spin $A A^{\prime} X X^{\prime}$ do complexo 6, no qual $A, A^{\prime}={ }^{31} P$ e $X, X^{\prime}={ }^{195} P t$.

\section{RMN ${ }^{195}$ PT DE SÓLIDOS}

A quantidade de trabalhos envolvendo $\mathrm{RMN}{ }^{195} \mathrm{Pt}$ de sólidos é bem pequena. Uma extensa faixa de deslocamentos químicos e efeitos anisotrópicos bem pronunciados em complexos quadrados de $\mathrm{Pt}(\mathrm{II})$ (anisotropias em torno de $10.000 \mathrm{ppm}$ ) são as principais causas do pouco interesse em RMN ${ }^{195} \mathrm{Pt}$ de sólidos ${ }^{42}$.

Os deslocamentos químicos, como já foi dito, dependem da blindagem que envolve cada núcleo. $\mathrm{O}$ tensor de blindagem $\sigma$ é composto basicamente por três componentes: $\sigma_{11}, \sigma_{22}$ e $\sigma_{33}$ (outros componentes têm um efeito negligenciável no espectro). A média aritmética entre eles é chamada de tensor isotrópico $\sigma_{\text {iso. }}$ Os deslocamentos químicos de núcleos em espectros de soluções provêm dos tensores isotrópicos $\sigma_{\text {iso. }}{ }^{2}$.

Em sólidos, um fator que muito contribui para a largura do sinal é o deslocamento químico anisotrópico, que se origina 
das várias orientações das moléculas de um composto policristalino em relação ao campo magnético $\mathbf{B}_{\mathbf{0}}$. Uma das orientações possíveis contribuiria para uma blindagem máxima do núcleo estudado e o deslocamento químico estaria em uma freqüência mínima. Uma outra possibilidade de posição contribuiria para uma blindagem mínima, com o deslocamento químico em uma freqüência máxima. A largura do sinal seria determinada por estes deslocamentos químicos. Para um monocristal, seria observado apenas um sinal fino com deslocamento químico variável com a orientação do cristal no campo magnético ${ }^{43}$.

$\mathrm{O}$ espectro de $\mathrm{RMN}{ }^{195} \mathrm{Pt}$ de sólido do complexo $\left[\mathrm{PtMe}_{3}(\mathrm{acac})\right]_{2}(\mathrm{acac}=$ acetilacetonato $)$ mostra um sinal largo, no qual podemos notar os componentes principais do tensor de blindagem e o tensor isotrópico (Fig. 14). A anisotropia ${ }^{44}$ deste complexo é relativamente grande: $\Delta \sigma=1123 \mathrm{ppm}$. Complexos octaédricos de $\mathrm{Pt}(\mathrm{IV})$ costumam ter anisotropias abaixo de $1000 \mathrm{ppm}$, enquanto complexos quadrados de platina (II) apresentam anisotropias próximas de 10.000 ppm (um octaedro é "mais entérico" que um quadrado) $)^{45,46}$.

Outro fator de anisotropia é o acoplamento direto D (acopla-

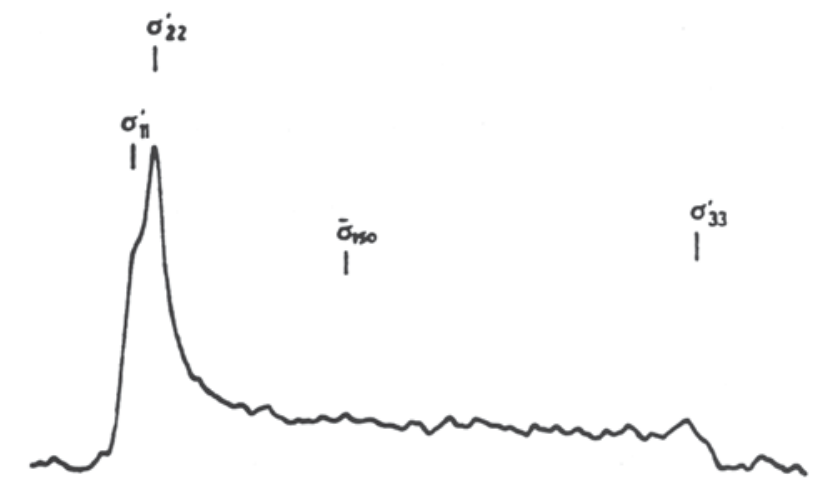

Figura 14. Espectro de $R M N{ }^{195}$ Pt no estado sólido de $\left[\mathrm{PtMe}{ }_{3}(\mathrm{acac})\right]_{2}$ (ref. 45).

mento dipolo-dipolo), que deriva da interação dipolar direta entre dois momentos magnéticos nucleares através do espaço. O desdobramento da ressonância de um núcleo $X$, ocasionado pelo acoplamento dipolo-dipolo com outro núcleo $\mathrm{Y}$, depende das constantes giromagnéticas $\gamma_{\mathrm{X}}$ e $\gamma_{\mathrm{Y}}$, da distância $r$ entre os núcleos $\mathrm{X}$ e Y, e do ângulo $\theta$ entre o campo $\mathbf{B}_{\mathbf{0}}$ e o vetor $\mathbf{r}$, que une os núcleos $\mathrm{X}$ e $\mathrm{Y}$ :

$\mathrm{D} \propto \gamma_{\mathrm{X}} \cdot \gamma_{\mathrm{Y}} \cdot\left(3 \cos ^{2} \theta-1\right) / \mathrm{r}^{3}$

As interações dipolares não contribuem para as posições das linhas de ressonância em espectros de soluções. A movimentação das moléculas faz com que a soma de todas as orientações do vetor $\mathbf{r}$ em relação ao campo $\mathbf{B}_{0}$ seja nula ${ }^{2}$.

Valores de acoplamentos dipolares envolvendo ${ }^{1} \mathrm{H}$ são muito grandes devido à sua constante giromagnética. Estes acoplamentos normalmente inviabilizam o espectro, sendo necessária a utilização de desacopladores de alta potência ${ }^{43}$.

Os acoplamentos escalares ou indiretos $J$ podem também ser observados em espectros no estado sólido. São independentes da anisotropia molecular (a transmissão do acoplamento se dá através dos elétrons das ligações químicas).

Uma maneira de amenizar os fatores anisotrópicos consiste na utilização da técnica MAS (do inglês: Magic Angle Spinning). Nesta técnica, a amostra sofre alta rotação com um ângulo de $54,7^{\circ}$ (ângulo mágico) em relação ao campo magnético ${ }^{47}$. Quando a rotação da amostra não é suficiente para neutralizar totalmente os fatores anisotrópicos, observam-se bandas laterais. Os espectros, estático e com rotações de 3200 $\mathrm{Hz}$ e $1907 \mathrm{~Hz}$, do composto $\mathrm{K}_{2}\left[\mathrm{Pt}(\mathrm{OH})_{6}\right]$ mostram que o número de bandas laterais decresce com o aumento da frequiência de rotação da amostra (Fig. 15) ${ }^{46}$. A diferenciação entre bandas laterais e sinais de ressonância é feita comparando-se os espectros obtidos em duas diferentes freqüências de rotação da amostra. As bandas laterais se modificam com a variação da frequiência de rotação, enquanto os deslocamentos químicos dos sinais da amostra são mantidos.

Outro fator importante a ser comentado, é a questão da equivalência química entre núcleos no estado sólido. Núcleos que são quimicamente equivalentes em uma molécula em solução nem sempre são equivalentes no estado sólido. A molécula em sua cela unitária pode conter estes núcleos em sítios cristalográficos diferentes. Isto ocorre, por exemplo, com o complexo cis-[ $\left.\mathrm{PtMe}_{2}\left(\mathrm{PPh}_{3}\right)_{2}\right]$. O valor de $\delta(\mathrm{Pt})$ é único em $-4682 \mathrm{ppm}$ com os átomos de platina ocupando apenas um tipo de sítio cristalográfico. Mas há um dubleto de dubletos proveniente de duas constantes de acoplamentos distintas entre platina e fósforo: ${ }^{1} J\left(\mathrm{PtP}^{\mathrm{A}}\right)=1825 \mathrm{~Hz}$ e ${ }^{1} J\left(\mathrm{PtP}^{\mathrm{B}}\right)=1979 \mathrm{~Hz}$ (Fig. 16). Portanto, as duas fosfinas do complexo, estão em sítios cristalográficos diferentes, ou seja, não são quimicamente equivalentes no estado sólido $^{48}$.

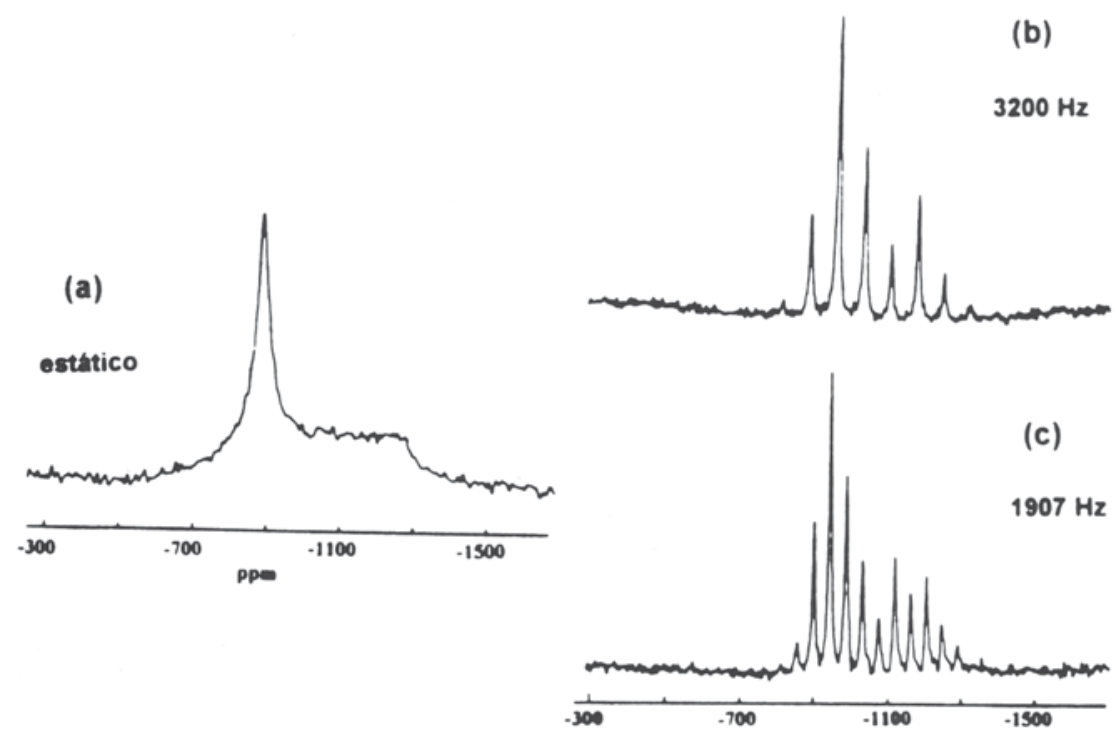

Figura 15. Espectro de RMN ${ }^{195} \mathrm{Pt}$ no estado sólido composto $\mathrm{K}_{2}\left[\mathrm{Pt}(\mathrm{OH})_{6}\right]$ : a) estático; b) com rotação de $3200 \mathrm{~Hz}$; c) com rotação de $1907 \mathrm{~Hz}$ (ref. 46). 


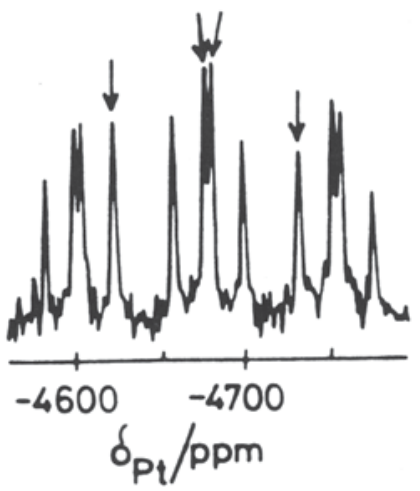

Figura 16. Espectro de RMN ${ }^{195} \mathrm{Pt}$ CP/MAS a 42,8 MHz de cis[PtMe $\left.2\left(\mathrm{PPh}_{3}\right)_{2}\right]$. As flechas indicam o dubleto de dubletos resultante dos acoplamentos ${ }^{1} J\left(P t P^{A}\right)=1825 \mathrm{~Hz} e^{1} J\left(P t P^{B}\right)=1979 \mathrm{~Hz}($ ref 48).

\section{CONSIDERAÇÕES FINAIS}

Espectros de RMN ${ }^{195} \mathrm{Pt}$ em solução estão se tornando rotineiros. Uma grande variedade de deslocamentos químicos e constantes de acoplamentos de várias espécies químicas contendo platina está documentada. Até o momento, esta técnica é mais empregada para caracterização de compostos de coordenação, principalmente os que contêm ligações entre átomos de platina e átomos de nitrogênio, oxigênio e cloro. Em comparação aos compostos de coordenação, poucos estudos têm sido realizados com compostos organometálicos de platina (com ligações Pt-C). Novos métodos, que utilizam dupla e tripla ressonância, têm sido desenvolvidos, ampliando as aplicações da RMN ${ }^{195} \mathrm{Pt}$. A RMN ${ }^{195} \mathrm{Pt}$ no estado sólido permite estudos de compostos sem influência do solvente ou da troca de ligantes, e de materiais insolúveis ou que não retenham a estrutura em solução. Provavelmente, o desenvolvimento desta técnica minimizará os efeitos das elevadas anisotropias dos complexos quadrados de platina(II), propiciando um aumento significativo de trabalhos nesta área.

\section{AGRADECIMENTOS}

Aos professores Gilson Herbert Magalhães Dias, Maria Domingues Vargas e Roberto Rittner Neto do Instituto de Química da UNICAMP pelas sugestões e incentivo.

\section{REFERÊNCIAS E NOTAS}

1. Pregosin, P. S.; Coord. Chem. Rev. 1982, 4, 4247.

2. Jameson, C. J.; Mason, J.; In Multinuclear NMR; Mason, J., Ed.; Plenum Press; Nova York, 1987; p 3.

3. Sanders, J. K. M.; Hunter, B. K.; Modern NMR Spectroscopy: A Guide for Chemists; Oxford University Press; Oxford, 1987; p 9.

4. Bovey, F. A.; Nuclear Magnetic Resonance Spectroscopy; Academic Press; Nova York, 1969; p 5.

5. Breitmaier, E.; Voelter, W.; Carbon-13 NMR Spectroscopy; VCH; Weinheim, $3^{\mathrm{a}}$ ed.; 1987; p 12.

6. Gil, V. M. S.; Geraldes, C. F. G. C.; Ressonância Magnética Nuclear: Fundamentos, Métodos e Aplicações; Fundação Calouste Gulbenkian; Lisboa, 1987.

7. Weeb, G. A.; In NMR and the Periodic Table; Harris, R. K.; Mann, B. E., Ed.; Academic Press; Nova York, 1978; p 75.

8. Howarth, O.; In Multinuclear NMR; Mason, J., Ed.; Plenum Press; Nova York, 1987; p 150.

9. Pregosin, P. S.; Annu. Rep. NMR Spectrosc. 1986, 17, 285.

10. Duvigneau, J.; Peters, G.; Preetz, W.; Z. Anorg. Allg. Chem. 1993, 619, 2043.

11. Multinuclear NMR; Mason, J., Ed.; Plenum Press; Nova York, 1987; p 625 (apêndice).

12. Clark, H. C.; Nicholas, A. M. P.; Magn. Reson. Chem. 1990, 28, 99.

13. Kidd, R. G.; Goodfellow, R. J.; In NMR anel the Periodic
Table, Harris, R. K.; Mann, B. E., Ed.; Academic Press; Londres, 1978; p 249.

14. Brown, C.; Heaton, B. J.; Sabounchei, J.; J. Organornet. Chem. 1977, 142, 413.

15. Goggin, P. L.; Goodfellow, R. J.; Haddock, S. R.; Taylor, B. F.; Marshall, I. R. H.; J. Chem. Soc., Dalton Trans. 1976, 459.

16. Kennedy, J. D.; McFarlane, W.; Puddephatt, R. J.; Thompson, P. J.; J. Chem. Soc., Dalton Trans. 1976, 874.

17. Benn, R.; Reinhardt, R. D.; e Rufinska, A.; J. Orgartomet. Chem. 1985, 282, 291.

18. Boardman, L. D.; Newmark R. A.; Magn. Reson. Chem. 1992, 30, 481 .

19. Brevard, C.; Granger, P.; Handbook of High Resolution Multinuclear NMR; Wiley; Nova York, 1981.

20. Georgii, I.; Mann, B. E.; Taylor, B. F.; Musco, A.; Inorg. Chim. Acta 1984, 86, L81.

21. Costuma-se representar o acoplamento entre dois núcleos, $\mathrm{A}$ e $\mathrm{B}$, como ${ }^{\mathrm{n}} J(\mathrm{AB})$, sendo que n é o número de ligações separando A de B.

22. Isotopômeros de um composto são moléculas idênticas com isótopos diferentes, por exemplo: $\mathrm{CHCl}_{3}$ e $\mathrm{CDCl}_{3}$ são dois isotopômeros do clorofórmio.

23. Ursini, C. V.; Tese de doutorado em andamento no Instituto de Química da Universidade Estadual de Campinas.

24. Sanders, J. K. M.; Hunter, B. K.; Modern NMR Spectroscopy: A Guide for Chemists; Oxford University Press; Oxford, 1987; p 179.

25. Nicolaides, A.; Smith, J. M.; Kumar, A.; Barnhart, D. M.; Borden, W. T.; Organometallics 1995, 14, 3475.

26. Yamamoto, A.; Organotransition Metal Chemistry: Fundamental Concepts and Applications; Wiley; Nova York, 1986; p 179.

27. Parish, R. V.; NMR, NQR, EPR, and Mössbauer Spectroscopy in Inorganic Chemistry; Ellis Horwood Limited; Chichester-Inglaterra, 1990; p 56.

28. Boag, N. M.; Green, M.; Grove, D. M.; Howard, J. A. K.; Spencer, J. L.; Stone, F. G. A.; J. Chem. Soc., Dalton Trans. 1980, 2170.

29. Cross, R. J.; Gemmill, J.; J. Chem. Soc., Dalton Trans. 1984, 199.

30. Green, M.; Howard, J. A. K.; Spencer, J. L.; Stone, F. G. A.; J. Chem. Soc., Dalton Trans. 1977, 271.

31. Goodfellow, R. J.; In NMR and the Periodic Table; Harris, R. K.; Mann, B. E., Ed.; Academic Press; Nova York, 1978; p 256.

32. Verkade, J. G.; Mosbo, J. A.; In Phosphorus-31 NMR Spectroscopy in Stereochemical Analysis: Organic Compounds and Metal Complexes; Verkade, J. G.; Quin, L. D.; Ed; VCH; Deerfield Beach-Florida, 1987; p 438.

33. Parzich, E.; Peters, G.; Preetz, W.; Z. Naturforsch. 1993, $48 b, 1169$.

34. Allevi, C.; Bonfà, M.; Garlaschelli, L.; Malatesta, M. C.; Gazz. Chim. Ital. 1990, 120, 711.

35. Becker, E. D.; High Resolution NMR: Theory and Chemical Applications; Academic Press; Nova York, $1969 ;$ p 89.

36. Sanders, J. K. M.; Hunter, B. K.; Modern NMR Spectroscopy: A Guide for Chemists; Oxford University Press; Oxford, 1987; p 217.

37. Grossel, M. C.; Batson, J. R.; Moulding, R. P.; Seddon, K. R.; J. Organomet. Chem. 1986, 304, 391.

38. Green, M.; Howard, J. A. K.; Murray, M.; Spencer, J. L.; Stone, F. G. A.; J. Chem. Soc., Dalton Trans. 1977, 1509.

39. Rüegger, H.; Moskau, D.; Magn. Reson. Chem. 1991, 29, S11.

40. Ursini, C. V.; Dias, G. H. M.; Gambardella, M. T. P.; Santos, R. H. A.; submetido à publicação. 
41. Becker, E. D.; High Resolution NMR: Theory anel Chemical Applicalions; Academic Press; Nova York, 1969; p 166.

42. Austin, E. J. W.; Barrie, P. J.; Clark, R. J. H.; J. Chem. Soc., Chem. Commun. 1993, 1404.

43. Sanders, J. K. M.; Hunter, B. K.; Modern NMR Spectroscopy: A Guide for Chemists; Oxford University Press; Oxford, 1987; p 260.

44. A anisotropia é definida como $\Delta \sigma=\sigma_{33}-\left(\sigma_{22}+\sigma_{11}\right) / 2$, sendo que, convencionalmente, $\sigma_{33} \geq \sigma_{22} \geq \sigma_{11}$. Jameson, C. J.; Mason, J.; In Multinuclear NMR; Mason, J., Ed.;
Plenum Press; Nova York, 1987; p 3.

45. Doddrell, D. M.; Barron, P. F.; Clegg, D. E.; Bowie, C.; J. Chem. Soc., Chem. Commun. 1982, 575.

46. Harris, R. K.; Reams, P.; Packer, K. J.; J. Chem. Soc., Dalton Trans. 1986, 1015.

47. Parish, R. V.; NMR, NQR, EPR, and Mössbauer Spectroscopy in Inorganic Chemistry; Ellis Horwood Limited; Chichester-Inglaterra, 1990; p 26.

48. Harris, R. K.; McNaught, I. J.; Reams, P.; Packer, K. J.; Magn. Reson. Chem. 1991, 29, S60. 